\title{
Expression of the adipocytokine resistin and its association with the clinicopathological features and prognosis of pancreatic ductal adenocarcinoma
}

\author{
CHONG-YI JIANG ${ }^{1}$, WEI WANG ${ }^{1}$, YU-LEI YIN ${ }^{2}$, ZU-RONG YUAN $^{1}$ and LIU-BO WANG ${ }^{2}$ \\ Departments of ${ }^{1}$ Surgery and ${ }^{2}$ Pathology, Huadong Hospital, Fudan University, Shanghai 200040, P.R. China
}

Received March 23, 2012; Accepted July 10, 2012

DOI: $10.3892 / \mathrm{ol} .2012 .865$

\begin{abstract}
Fat tissue is viewed as an active endocrine organ that secretes a variety of bioactive substances. Resistin, an adipocyte-secreted factor, is thought to be closely related to obesity, insulin resistance and inflammation, the three most significant risk factors for the progression of pancreatic cancer. However, the association between resistin and pancreatic cancer is still unknown. In this study, pancreatic tumor samples from 45 patients with pancreatic ductal adenocarcinoma were analyzed with immunohistochemistry for the expression of resistin. The correlation between resistin expression and clinicopathological features and prognosis were evaluated. Resistin staining was observed in $48.9 \%$ (22 of 45) of the cases. Resistin expression was more frequent in poorly differentiated tumors ( 9 of $9,100 \%$ ) compared to moderately differentiated tumors (11 of 28, 39.3\%) and welldifferentiated tumors $(2$ of $8,25 \%)(\mathrm{p}<0.01)$. The incidence of resistin expression in patients with Japan Pancreas Society stages III-IV (18 of 27, 66.7\%) was significantly higher than in subjects with stages I-II (4 of 18, 22.2\%) ( $\mathrm{p}<0.01)$. Patients with resistin-stained tumors had significantly shorter relapsefree survival times (median, 9 months) than patients with negative tumors (median, 18 months) $(\mathrm{p}<0.05)$. In addition, multivariate analysis showed that resistin expression was an independent prognostic factor for relapse-free survival of patients with pancreatic ductal adenocarcinoma $(\mathrm{p}<0.05)$. These results demonstrate that resistin may influence the progression of pancreatic tumors and may be a useful predictor of relapse-free survival in patients with pancreatic ductal adenocarcinoma.
\end{abstract}

Correspondence to: Dr Chong-Yi Jiang, Department of Surgery, Huadong Hospital, Fudan University, No. 221 Yanan Road (West), Shanghai 200040, P.R. China

E-mail: jiangzhongyi9@hotmail.com

Key words: resistin, pancreatic ductal adenocarcinoma, adipocytokine

\section{Introduction}

Pancreatic cancer is the most lethal abdominal malignancy, with an annual mortality rate approximating its incidence (1). Obesity is an independent risk factor for the development and progression of pancreatic cancer (2). Obese mice developed larger pancreatic tumors, more metastases and showed greater mortality (3).

During the last two decades, fat tissue has been viewed not only as an energy storage depot but also as an active endocrine organ that secrets a variety of bioactive substances known as adipocytokines (4). Resistin, an adipocyte-secreted factor, was discovered in 2001 and proposed as a potential link between obesity, insulin resistance and inflammation $(5,6)$, the three most significant risk factors for pancreatic cancer $(3,7)$. The administration of recombinant resistin to diet-induced and genetically obese mice impaired glucose tolerance (4). Blocking resistin action improved blood sugar levels in obese mice but impaired glucose tolerance in healthy ones (4). Moreover, proinflammatory mediators strongly increased the expression of resistin in peripheral blood mononuclear cells, suggesting a role for resistin in the process of inflammation (8). Silswal et al incubated macrophages with recombinant resistin and found increased production of proinflammatory cytokines TNF- $\alpha$ and IL-12 (9). However, the association between resistin and pancreatic cancer is still unknown.

In the present study, we determined the expression of resistin in pancreatic ductal adenocarcinoma using immunohistochemical techniques and analyzed its correlation with the clinicopathological features and prognosis. We found that the positive expression of resistin was significantly related to poor differentiation of tumors, advanced Japan Pancreas Society (JPS) stage and short relapse-free survival of the patients. In addition, multivariate analysis showed that resistin expression was an independent prognostic factor for relapse-free survival in patients with pancreatic ductal adenocarcinoma.

\section{Patients and methods}

Patients and pathological specimens. Data on 45 patients, who underwent radical surgery for primary pancreatic cancer between 2006 and 2009 at Huadong hospital, China, were analyzed. Informed consent was obtained from each patient 
prior to the study. The study was approved by the ethics committee of Fudan University, Shanghai, China. These patients included 27 males and 18 females with a mean age of 62 years (range, 48-77). No patients received radiotherapy or chemotherapy prior to surgery. A pancreatoduodenectomy was performed on 30 patients for pancreatic head cancer. A distal or total pancreatectomy was performed on the other 15 patients for cancer in the pancreatic body or tail. The surgical specimens were routinely fixed with $10 \%$ formalin and embedded in paraffin. They were subsequently cut into sections and stained with hematoxylin and eosin (H\&E) for routine histopathological examination. Pathological examination was performed by two expert pathologists. All tissue specimens of the 45 cases were histologically diagnosed as ductal adenocarcinoma based on the WHO histological classification of tumors of the exocrine pancreas. All patients had tumor-free resection margins on microscopic examination of the surgical specimens. According to the JPS stage classification (10), the clinical stages of disease were I, II, III and IV in 4, 14, 21 and 6 patients, respectively.

Immunohistochemistry. Staining for resistin protein was carried out using a rabbit polyclonal anti-resistin antibody (R1779, Sigma, St. Louis, MO, USA). Sections were deparafinized and pretreated with microwaves (15 min at $900 \mathrm{~W}$ ) for antigen retrieval. Next, the sections were treated with hydrogen peroxide for $5 \mathrm{~min}$ to stop endogenous peroxidase activity. Subsequently, these sections were incubated with the primary antibody (diluted to $1: 200$ ) at $4^{\circ} \mathrm{C}$ overnight and then incubated with a dextran polymer reagent combined with secondary antibodies and peroxidase (K5007, Dako, Denmark) for $2 \mathrm{~h}$ at room temperature. 3,3'-Diaminobenzidine (DAB) was used as the chromogen. Phosphate-buffered saline (PBS) was substituted for the primary antibody as a negative control.

Evaluation of immunohistochemical staining. Two expert pathologists independently evaluated the results from the immunohistochemical staining without any prior knowledge of patients' clinical data. To evaluate resistin expression, a score was established corresponding to the sum of: a) the percentage of positive cells $(0,0 \%$ immunopositive cells; $1,<25 \%$ positive cells; $2,26-50 \%$ positive cells; and $3,>50 \%$ positive cells); and b) the staining intensity ( 0 , negative; 1 , weak; 2 , moderate; 3 , high). The sum of $a+b$ reached a maximum score at 6 . Scores between 0 and 2 were regarded as negative, and scores between 3 and 6 as positive (11).

Follow-up. The follow-up was performed in our institution on an outpatient basis. Patients were followed up at least quarterly for 2 years, then at least biannually. Recurrence was diagnosed based on computed tomography cross-sectional imaging. The survival times were determined from the day of surgery with follow-up to February 2012 (median follow-up, 22 months). One patient was lost to follow-up 6 months after surgery.

Statistical analysis. All statistical analyses were performed using SPSS version 12.0 for Windows (Chicago, IL, USA). For the statistical analyses, the t-test and Chi-square test were used.
Table I. Patients with positive resistin expression compared with the subjects with negative expression by age and BMI.

\begin{tabular}{lccc}
\hline & \multicolumn{2}{c}{ Resistin expression (mean $\pm \mathrm{SD})$} & \\
\cline { 2 - 3 } & $\begin{array}{c}\text { Positive } \\
(\mathrm{n}=22)\end{array}$ & $\begin{array}{c}\text { Negative } \\
(\mathrm{n}=23)\end{array}$ & Significance $^{\mathrm{a}}$ \\
\hline Age $(\mathrm{years})$ & $62.7 \pm 8.8$ & $61.6 \pm 7.1$ & 0.314 \\
BMI $\left(\mathrm{kg} / \mathrm{m}^{2}\right)$ & $22.4 \pm 2.8$ & $21.6 \pm 2.7$ & 0.746 \\
\hline
\end{tabular}

at-test.

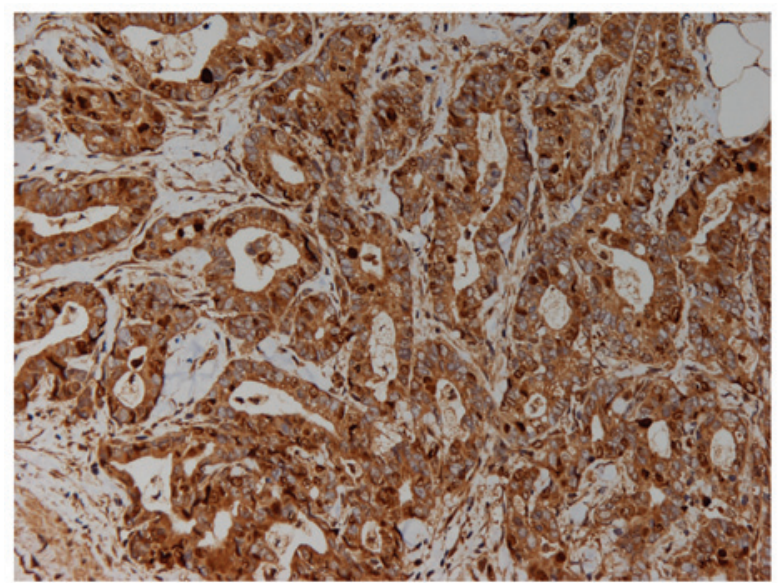

Figure 1. Positive expression of resistin in pancreatic ductal adenocarcinoma tissue (immunohistochemical staining, x200). The cytoplasm was heavily stained.

Life-table analyses, according to the Kaplan-Meier method, were performed for overall survival and relapse-free survival. Differences in survival between subgroups were compared with the log-rank test. To define independent risk factors for prognosis, multivariate analysis was performed with a Cox proportional hazards model. $\mathrm{P}<0.05$ was considered to indicate a statistically significant result.

\section{Results}

Resistin staining was found in 22 of the 45 tumors (48.9\%) analyzed (Fig. 1). The patients with positive resistin expression were matched to those with negative expression by age and body mass index (BMI) (Table I). The correlation between resistin expression and the clinicopathological features is shown in Table II. Resistin expression was observed in $100 \%$ (9 of 9) of poorly differentiated tumors, 39.3\% (11 of 28 ) of moderately differentiated tumors, and $25 \%$ (2 of 8 ) of well-differentiated tumors $(\mathrm{p}<0.01)$. The incidence of resistin expression in patients with JPS stages III-IV (66.7\%, 18 of 27) was significantly higher than in those with stages I-II $(22.2 \%$, 4 of 18$)(p<0.01)$. In addition, resistin expression was more frequent in patients with neural invasion $(53.7 \%, 22$ of 41$)$ 
Table II. Correlation between resistin expression and clinicopathological features.

\section{Expression of resistin}

Clinicopathological features

n

Positive $(\%)$

Negative (\%)

Significance

\section{Gender}

Male 27

Female

$14(51.9)$

$9(50.0)$

$9(50.0)$

0.903

Tumor area

Head

30

$14(46.7)$

$16(53.3)$

Body and/or tail

7 (46.7)

Tumor size $(\mathrm{cm})$

$\begin{array}{ll}\leq 2 & 10 \\ >2 & 35\end{array}$

$10 \quad 6(60.0)$

$4(40.0)$

Lymph node metastasis

Positive 16

Negative

Tumor differentiation

Poorly differentiated

Moderately differentiated

$14(48.3)$

15 (51.7)

Well-differentiated

JPS stage

\begin{tabular}{lccr} 
I-II & 18 & $4(22.2)$ & $14(77.8)$ \\
III-IV & 27 & $18(66.7)$ & $9(33.3)$ \\
Neural invasion & & & \\
Positive & 41 & $22(53.7)$ & $19(46.3)$ \\
Negative & 4 & $0(0)$ & $4(100)$ \\
\hline
\end{tabular}

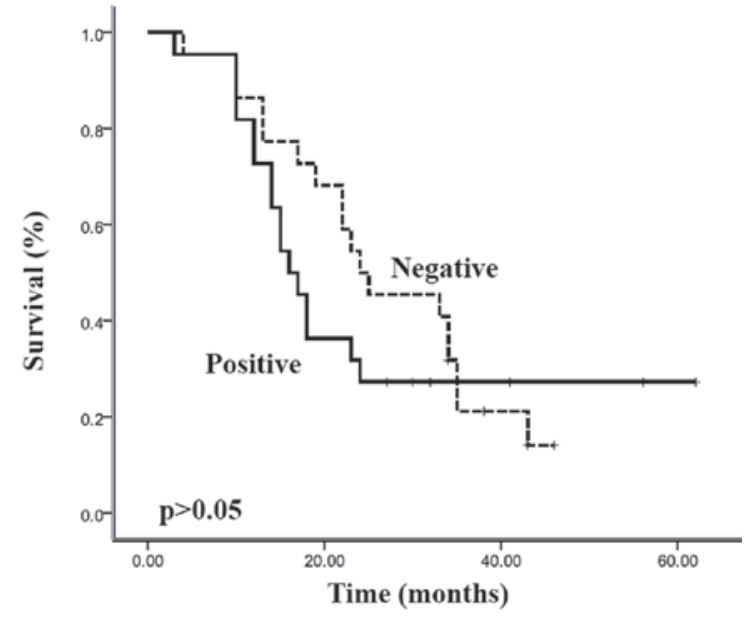

Figure 2. Overall survival curves of patients with pancreatic ductal adenocarcinoma (Kaplan-Meier estimates), grouped according to expression of resistin (positive/negative). There was no significant correlation between resistin expression and overall survival ( $p>0.05)$.

than in subjects without neural invasion $(0 \%, 0$ of 4$)$, but this difference did not reach statistical significance $(\mathrm{p}>0.05)$.

Complete follow-up was available for $97.8 \%$ (44 of 45) of patients in this study. The overall survival rate of the entire cohort was $80 \%$ at 1 year, $22 \%$ at 3 years and $17 \%$ at 5 years,

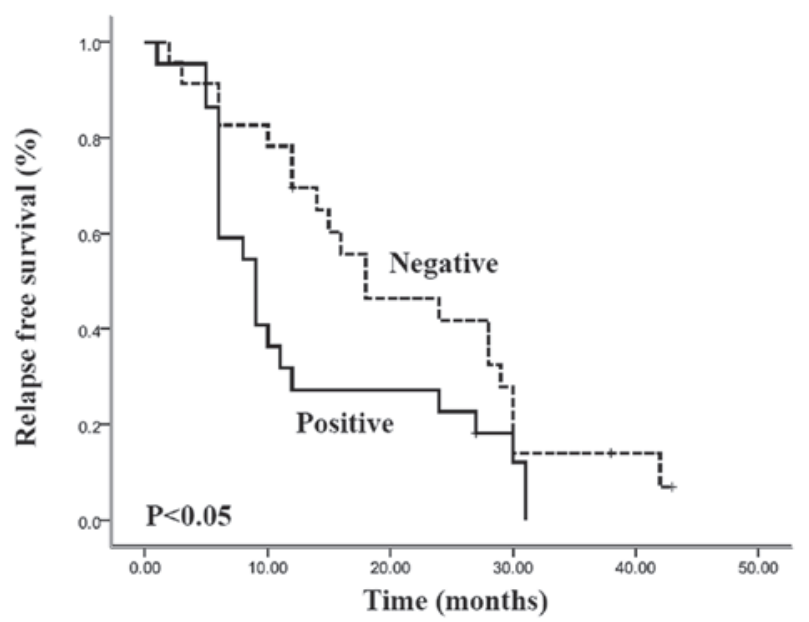

Figure 3. Relapse-free survival curves of patients with pancreatic ductal adenocarcinoma (Kaplan-Meier estimates), grouped according to expression of resistin (positive/negative). Relapse-free survival of resistin-positive patients was shorter than the patients with resistin-negative expression, and there was a statistically significant difference in survival curves between these two groups $(\mathrm{p}<0.05)$.

with a median survival of 22.0 months [ $95 \%$ confidence interval (CI), 16.4-27.6 months]. The overall survival and relapse-free survival were stratified according to the resistin expression, 
and the results are shown in Figs. 2 and 3, respectively. Patients with positive resistin expression had a shorter overall survival (median 16.0 months; 95\% CI, 12.9-19.1 months) than the patients with negative resistin expression (median 24.0 months; 95\% CI, 11.4-36.6 months). However, Kaplan-Meier curves showed there was no significant correlation between resistin expression and overall survival $(\mathrm{p}>0.05)$. Relapse-free survival of patients with resistin-positive expression (median, 9.0 months; 95\% CI, 5.6-12.4 months) was also shorter than that of patients with resistin-negative expression (median, 18.0 months; 95\% CI 6.0-30.0 months), and there was a statistically significant difference in the survival curves between these two groups $(\mathrm{p}<0.05)$. In addition, the multivariate analysis (Cox regression model) clearly demonstrated that resistin expression was an independent prognostic factor for the relapse-free survival of patients with pancreatic ductal adenocarcinoma $(\mathrm{p}<0.05)$.

\section{Discussion}

The incidence of pancreatic cancer, particularly ductal adenocarcinoma, is increasing in the Western world and China. Due to its poor prognosis, pancreatic cancer has become the most lethal abdominal malignancy with a five-year mortality rate of over $95 \%$, and a median survival rate of 3 to 6 months from diagnosis (1). Radical resection is the most effective therapy to improve the survival rate of patients with pancreatic cancer (12). In this paper, the overall survival of the entire cohort who underwent radical surgery was $80 \%$ at 1 year, $22 \%$ at 3 years and $17 \%$ at 5 years, with a median survival of 22 months. These results are similar to those of other studies $(12,13)$.

The adipocytokine resistin is closely related to obesity, insulin resistance and systemic proinflammatory response $(4,14,15)$, all of which are recognized as significant factors involved in the growth and metastasis of pancreatic cancer (16-18). Using a murine model of obesity, Zyromski et al (3) demonstrated that insulin resistance could lead directly to changes in the tumor microenvironment, thereby promoting pancreatic cancer growth and dissemination. Chronic inflammation is known to be one of the causes of cancer development (18). Proinflammatory stimuli stimulate stromal fibroblasts to produce growth factors, initiate malignant cell growth, inhibit apoptosis, inhibit cytotoxic T-lymphocyte activity, promote angiogenesis, facilitate metastasis and enhance tumor-associated macrophage infiltration $(18,19)$. Patients with chronic pancreatitis have a $10-$ to 20-fold increased risk of developing pancreatic cancer (20).

To our knowledge, this is the first study to date to evaluate the correlation between the adipocytokine resistin and pancreatic ductal adenocarcinoma. The present study established a strong correlation between resistin expression, poor differentiation of tumors, advanced JPS stage and patient outcome. An independent role of resistin in pancreatic ductal adenocarcinoma progression was confirmed by the Cox multivariate analysis in which positive resistin expression was a significant prognosticator for relapse-free survival. Taken together, these results predict that resistin may influence the progression of pancreatic ductal adenocarcinoma. Consistent with our results, resistin has been shown to play a key role in promoting tumorigenesis in several studies $(21,22)$. In choriocarcinoma, resistin may induce the expression of matrix metalloproteinases (MMPs), reduce the synthesis of tissue inhibitors of metalloproteinases (TIMP), and thereby increase the invasiveness of trophoblast-like cells (21). Resistin has also been shown to induce production of vascular endothelial growth factor receptor (VEGFR) and the formation of endothelial cell tubes, thus facilitating tumorigenesis (22).

There are several limitations of this study. The number of study subjects was limited and the risk factors of pancreatic cancer such as smoking and history of pancreatitis were not considered. A large-scale prospective study of the precise mechanisms of the association of resistin with pancreatic cancer should be supported in the future.

In conclusion, this study demonstrates that pancreatic cancer patients with resistin-positive expression have a poorer histological grade, a higher advanced stage (JPS stage III-IV) and a shorter relapse-free survival. These results suggest that resistin may influence the progression of pancreatic tumors and may be a good prognostic indicator for relapse-free survival of patients with pancreatic ductal adenocarcinoma.

\section{Acknowledgements}

This study was supported by grants from the National Natural Science Foundation of China (NSFC, No. 30901433) and the Shanghai Shenkang Hospital Development Center (No. SHDC12010120).

\section{References}

1. Jemal A, Siegel R, Xu J and Ward E: Cancer statistics, 2010. CA Cancer J Clin 60: 277-300, 2010.

2. Calle EE, Rodriguez $\mathrm{C}$, Walker-Thurmond $\mathrm{K}$ and Thun MJ: Overweight, obesity, and mortality from cancer in a prospectively studied cohort of U.S. adults. N Engl J Med 348: 1625-1638, 2003.

3. Zyromski NJ, Mathur A, Pitt HA, et al: Obesity potentiates the growth and dissemination of pancreatic cancer. Surgery 146: 258-263, 2009.

4. Steppan CM, Bailey ST, Bhat S, et al: The hormone resistin links obesity to diabetes. Nature 409: 307-312, 2001.

5. Filkova M, Haluzik M, Gay S and Senolt L: The role of resistin as a regulator of inflammation: Implications for various human pathologies. Clin Immunol 133: 157-170, 2009.

6. McTernan PG, Kusminski CM and Kumar S: Resistin. Curr Opin Lipidol 17: 170-175, 2006.

7. Gumbs AA: Obesity, pancreatitis, and pancreatic cancer. Obes Surg 18: 1183-1187, 2008.

8. Bokarewa M, Nagaev I, Dahlberg L, Smith U and Tarkowski A: Resistin, an adipokine with potent proinflammatory properties. J Immunol 174: 5789-5795, 2005.

9. Silswal N, Singh AK, Aruna B, Mukhopadhyay S, Ghosh S and Ehtesham NZ: Human resistin stimulates the pro-inflammatory cytokines TNF-alpha and IL-12 in macrophages by NF-kappaBdependent pathway. Biochem Biophys Res Commun 334: 1092-1101, 2005

10. Isaji S, Kawarada Y and Uemoto S: Classification of pancreatic cancer: comparison of Japanese and UICC classifications. Pancreas 28: 231-234, 2004.

11. Volm M, Koomagi R and Mattern J: Prognostic value of vascular endothelial growth factor and its receptor Flt-1 in squamous cell lung cancer. Int J Cancer 74: 64-68, 1997.

12. Cameron JL, Riall TS, Coleman J and Belcher KA: One thousand consecutive pancreaticoduodenectomies. Ann Surg 244: 10-15, 2006.

13. Sohn TA, Yeo CJ, Cameron JL, et al: Resected adenocarcinoma of the pancreas-616 patients: results, outcomes, and prognostic indicators. J Gastrointest Surg 4: 567-579, 2000. 
14. Qasim AN, Metkus TS, Tadesse M, et al: Resistin gene variation is associated with systemic inflammation but not plasma adipokine levels, metabolic syndrome or coronary atherosclerosis in nondiabetic Caucasians. Clin Endocrinol (Oxf) 70: 698-705, 2009.

15. Bertolani C, Sancho-Bru P, Failli P, et al: Resistin as an intrahepatic cytokine: overexpression during chronic injury and induction of proinflammatory actions in hepatic stellate cells. Am J Pathol 169: 2042-2053, 2006.

16. Pollak M: Insulin-like growth factor-related signaling and cancer development. Recent Results Cancer Res 174: 49-53, 2007.

17. Stolzenberg-Solomon RZ, Graubard BI, Chari S, et al: Insulin, glucose, insulin resistance, and pancreatic cancer in male smokers. JAMA 294: 2872-2878, 2005.

18. Coussens LM and Werb Z: Inflammation and cancer. Nature 420 $860-867,2002$.
19. Zhang Z and Rigas B: NF-kappaB, inflammation and pancreatic carcinogenesis: NF-kappaB as a chemoprevention target (review). Int J Oncol 29: 185-192, 2006.

20. Howes N and Neoptolemos JP: Risk of pancreatic ductal adenocarcinoma in chronic pancreatitis. Gut 51: 765-766, 2002.

21. Di Simone N, Di Nicuolo F, Sanguinetti M, et al: Resistin regulates human choriocarcinoma cell invasive behaviour and endothelial cell angiogenic processes. J Endocrinol 189: 691-699, 2006.

22. Mu H, Ohashi R, Yan S, et al: Adipokine resistin promotes in vitro angiogenesis of human endothelial cells. Cardiovasc Res 70: 146-157, 2006. 